\title{
Digital infrared breast scan shows promise for detecting cancer
}

In the UK, 45,000 women are diagnosed each year with breast cancer, and early detection is paramount to improve survival rates. Screening mammography has resulted in a $15-20 \%$ reduction in breast cancer mortality, and cancers detected by this method are likely to be smaller, and well differentiated.

Mammography is performed every 3 years in women aged $50-70$ years in the UK. Despite the benefit of mammography, the sensitivity of detection of disease is low in younger women owing to the difficulty of imaging dense breast tissue. Now, a study has shown that imaging with a digital infrared breast (DIB) scan is an effective detection method in women under 70 years and shows particular promise in women under the age of 50 .

Wishart and colleagues assessed the effectiveness of DIB imaging in 106 biopsies taken from 100 women; of these, 65 were malignant and 41 were benign.
The DIB technique was most effective for detecting tumors in women younger than 50 years, with a sensitivity of $78 \%$ and a specificity of $75 \%$. In women under 50 years of age, this technique had a similar sensitivity to mammography (78\%). When both mammography and DIB were combined the sensitivity increased to $89 \%$.

"The results of this study support the use of DIB as an effective adjunctive test for breast cancer detection in women under 70 years of age. DIB appears to be particularly effective in women under 50 years old," Wishart concludes. Further studies are aimed at exploring how DIB can help detect and monitor breast cancer in response to neoadjuvant therapies.

\footnotetext{
Lisa Hutchinson

Original article Wishart, G. C. et al. The accuracy of digital infrared imaging for breast cancer detection in women undergoing breast biopsy. Eur. J. Cancer Surg. 36, 535-540 (2010)
} 